\title{
Análisis de las estrategias de comunicación de las alcaldias de Circasia y Donmatías para la promoción y la prevención de salud pública durante la COVID-19 entre marzo y agosto de 2020
}

Analysis of the communication strategies
of the Municipalities of Circasia and
Donmatias for the promotion and
prevention of public health during
COVID-19 between March and August 2020

DOI: https://doi.org/10.18566/comunica.n46.a04

Recibido: 23 de julio de 2021

Aceptado: 3 de noviembre de 2021

\section{Resumen}

La pandemia de la COVID-19 desafía a los gobiernos nacional y locales en diferentes aspectos: económicos, sociales, culturales, la priorización de la salud pública y la preservación de la vida; por lo tanto, lograr credibilidad y captar la atención de los ciudadanos es un reto para las instituciones gubernamentales. El propósito fundamental de contener la propagación de la pandemia obliga a centralizar el manejo de la información y a plantear estrategias, tácticas y actividades de comunicación para gobernar y mantener la relación con las personas en esta situación de incertidumbre. En esta investigación se analizaron las estrategias de comunicación desarrolladas por las oficinas de comunicaciones de las alcaldías de Donmatías (Antioquia)
Comunicación

número 46

Enero-junio

2022 | pp. 48-70

\section{Daniela}

Osorno Tejada

Comunicadora de la Universidad de Antioquia. https://orcid.org/00000002-5770-5306 danielaosorn01020@gmail. com; daniela.osornot@ udea.edu.co

\section{María Fernanda Restrepo Uribe} Comunicadora de la Universidad de Antioquia. https://orcid.org/00000002-3759-5541 maferu1130@gmail.com; maria.restrepo34@udea. edu.co

\section{Juan Pablo} Rodríguez Martínez Comunicador de la Universidad de Antioquia. https://orcid.org/00000001-9681-679X

juanpablorm96@gmail.com; juan.rodriguezm1@udea. edu.co.

\section{María Helena Vivas López}

Comunicadora social de la Universidad de Antioquia. Especialista en Relaciones Industriales de la Universidad EAFIT. Magister en Filosofía Política de la Universidad de Antioquia. Actualmente, profesora de dicha universidad. https://orcid.org/00000002-2807-6977 maria.vivas@udea.edu.co 
y Circasia (Quindío), con el fin de entender cuáles fueron las lógicas de gobernanza y cuáles fueron los retos enfrentados en la gobernabilidad.

\section{Abstract}

The spread of COVID-19 pandemic challenges governments in cultural, social and economic aspects in which public health and preserving life must be matters to keep under control. For the official institutions the main goal is prevent the spread of coronavirus obligating to centralize information with which they create communication strategies, tactics and activities to govern and keep the relationship with their citizens in these uncertain times. This research analyzed communication strategies developed by the offices of communication in the majors of Donmatias and Circasia which make communication a key factor in their communities to face the challenges that arise in the public function, focused on the current health crisis.

\section{Introducción}

El 14 de marzo de 2020, el Gobierno nacional, por medio de la Resolución 000844, decretó la emergencia sanitaria en Colombia con el objetivo de prevenir y controlar la propagación del virus SARS Cov2, causante de la COVID-19, en el territorio nacional. Por esta razón, los gobiernos departamentales, locales y la sociedad civil debieron coadyuvar en la implementación de las normas, tal como lo establece el Manual de estrategias de información, educación y comunicación en salud, incluyente y accesible, publicado en el año 2014 y que señala la guía para crear una estrategia que permita informar al público sobre asuntos de salud, promover estilos de vida saludables y generar impactos en la población (Ministerio de Salud, 2018).

Entonces, esta situación ha llevado a crear estrategias que consideren los cambios y retos del día a día, como, por ejemplo, migrar hacia el mundo virtual. Agudelo (2016) rescata el hecho de reconocer la interacción que tiene la sociedad para construir y deconstruir mensajes en torno a la salud, con el fin de que el emisor reciba, resignifique, apropie y transforme sus acciones. Un aspecto relevante para el estudio es que los municipios de Donmatías y Circasia se encontraban en la formulación de los planes de desarrollo 20202023, los cuales fueron modificados para enfrentar la pandemia y debieron ser aprobados por canales digitales.

Esta contingencia ha afectado a muchos países, lo cual ha generado el desarrollo de la literatura en este campo. Autores como Aleixandre-Benavent
Comunicación

número 46

Enero-junio

2022

\section{Carlos Mario} Cano Ramírez

Psicólogo de la Universidad de Antioquia. Magíster en Ciencia Política de esa misma institución. PhD en Ciencias Humanas y Sociales de la Universidad Nacional de Colombia. Docente de Diseño de Vestuario en la Universidad Pontificia Bolivariana. https://orcid.org/00000002-0262-527X cibercultural@hotmail.com; carlos.cano@upb.edu.co.

\section{Palabras clave}

Comunicación en salud, Comunicación pública, COVID-19, Estrategias comunicativas, Salud pública.

\section{Keywords}

Health communication, Public communication, COVID-19, Communication strategies, Public health. 
et al. (2020) encaminaron sus esfuerzos en hablar de la importancia de la comunicación y la información en tiempos de pandemia por medio del análisis del flujo de aquella durante los primeros meses de cuarentena en España. Así, presentan el concepto de infodemia, haciendo referencia al exceso de información que circula sobre la COVID-19 y la dificultad para encontrar información fidedigna. Además, los autores hablan sobre las estrategias de comunicación usadas a partir de la experiencia vivida en Wuhan y España, y llegan a la conclusión de la necesidad de generar una comunicación abierta, honesta y frecuente desde los gobiernos.

Este, en particular, es un estudio de las estrategias de comunicación utilizadas durante la contingencia provocada por este tipo de coronavirus en las alcaldías de Circasia (Quindío) y Donmatías (Antioquia). Circasia, en el norte del Quindío, tiene 27.726 habitantes (Departamento Administrativo Nacional de Estadística [DANE], 2007) y es reconocido principalmente por el aporte que hace a su territorio con la producción cafetera. Donmatías, en el norte de Antioquia, tiene 22.243 habitantes (Departamento Administrativo Nacional de Estadística [DANE], 2015) y es conocido popularmente por su actividad económica como la fábrica de confección más grande de Colombia.

Los dos municipios están regidos por diferentes corrientes políticas: el municipio quindiano por el Partido Liberal y el municipio antioqueño por la coalición de los partidos ASI, U y Verde. Ambos municipios poseen un nivel demográfico similar.

Para esta investigación, la situación de casos positivos fue cambiante; en Donmatías, según reportes hechos en Facebook por la administración municipal, al inicio de la pandemia, a finales de marzo, había cero casos confirmados, pero 35 sospechosos; en cambio, para finales de agosto había 87 casos positivos y 395 finalizados. Según las cifras compartidas en la página web de la Gobernación del Quindío, en Circasia se empezaron a recibir informes de casos activos el 26 de marzo de 2020, dos en el municipio, y para el 31 de agosto se registraron 41 casos, con $0 \%$ de mortalidad.

Esta contingencia de salud pública se ha venido afrontando de diversas formas; por lo tanto, el propósito de esta investigación fue analizar las estrategias de comunicación de las alcaldías de Circasia y Donmatías para la promoción y la prevención durante el aislamiento preventivo obligatorio, entre marzo y agosto de 2020. Para hacer tal acercamiento, se describieron las estrategias de comunicación utilizadas, se determinaron los canales y medios de comunicación, se reconocieron los mensajes de las alcaldías para la promoción de hábitos necesarios y se examinó el grado de los mensajes de tal estrategia dirigidos a los ciudadanos, con el fin de analizar el sistema comunicativo en el que se desarrollaron. 


\section{Metodología}

Los objetivos fueron abordados con enfoque cualitativo, referenciado este principalmente por Galeano (2018) y base para analizar las estrategias de comunicación de estas alcaldías para la promoción y la prevención en salud pública. El objeto de estudio estuvo compuesto por diferentes cualidades y atributos del emisor: medios y canales de comunicación que utilizan ambas alcaldías y los diferentes mensajes que difundieron. Por otra parte, se estudió el proceso de recepción de la información, que en esta investigación se aborda como el grado de comprensión de los mensajes entre los ciudadanos.

Con el fin de comprender esta realidad social, a partir de los aspectos particulares de cada municipio y por medio de una visión cercana de lo que sucede con este fenómeno, fueron necesarios la perspectiva interpretativa y el paradigma fenomenológico, pues se trata de un proceso dialéctico y sistémico que incluye al sistema de comunicación y el sistema social, e integra sus componentes, entre ellos, los emisores y receptores.

Para cada objetivo se estableció un método con el fin de obtener los datos a partir de la indagación gracias a la participación de personas que tuvieron responsabilidades o acciones para llevar a cabo las estrategias de comunicación en cada alcaldía:

- Objetivo 1: Describir las estrategias de comunicación utilizadas por las alcaldías de Circasia y Donmatías durante el aislamiento preventivo obligatorio por la COVID-19 entre marzo y agosto de 2020.

- Método estudio de caso: Las alcaldías de Donmatías y Circasia son una muestra que ejemplifica cómo se desarrollan estrategias de comunicación en tiempo de crisis, para así poner estas en un contexto de pandemia.

- Instrumentos: Entrevistas semiestructuradas a los comunicadores de las alcaldías y funcionarios de las dependencias de salud y matriz de listado de estrategias.

- Objetivo 2: Determinar los canales y medios utilizados por las alcaldías de Circasia y Donmatías en el marco de las estrategias para la comunicación con los ciudadanos durante la contingencia provocada por la COVID-19 entre marzo y agosto de 2020.

- Método de revisión documental: Permite distinguir particularidades dentro de cada administración y así complementar el análisis determinando las descripciones precisas de lo que cada una de ellas realiza. 
- Instrumentos: Mapeo de las estrategias y los canales empleados en cada una de las administraciones a través de lo documentado en cada una de las entrevistas.

- Objetivo 3: Reconocer los mensajes que formaron parte de las estrategias utilizadas por las alcaldías de Circasia y Donmatías en la promoción de hábitos necesarios para prevenir la COVID-19 entre marzo y agosto de 2020.

- Método análisis de contenido: Permite descubrir la estructura interna de la comunicación (composición, organización, dinámica) y el contexto en el cual se produce la información (Galeano, 2018, p. 30).

- Instrumentos: Matriz que permite revisar y clasificar el contenido de ambas alcaldías.

- Objetivo 4: Examinar el grado de comprensión de los mensajes que formaron parte de las estrategias comunicativas dirigidas a los ciudadanos por parte de las alcaldías de Circasia y Donmatías durante la contingencia provocada por la COVID-19 entre marzo y agosto de 2020.

- Método análisis estadístico de la población: Permite organizar y sintetizar la información, ver sus características más relevantes y presentar la información de una manera concisa y clara, mediante gráficos y tablas de sencilla interpretación.

- Instrumentos: La herramienta Formularios, de Google, permitió condensar y sistematizar 14 preguntas, entre abiertas y cerradas, que permitieran comprender cómo han sido tomados los mensajes de las publicaciones emitidas por las alcaldías entre los diferentes públicos circasianos y donmatieños.

Galeano (2018) plantea que "para la realización de una investigación social cualitativa, no existe una estrategia estándar, lo característico es la confluencia de varias de ellas" (2018, p. 3). Por ello, en esta investigación se usaron instrumentos cualitativos y cuantitativos para recolectar datos e información relevante, y así desarrollar los objetivos propuestos con métodos diferenciados, como se verá más adelante. Para dar respuesta a los cuatro objetivos específicos, se creó igual número de matrices analíticas, las cuales fueron evolucionando para obtener una comprensión amplia del objeto de estudio.

Hallazgos y discusión: Se relatan los nueve hallazgos, analizados a la luz de diferentes autores y nuevas teorías empleadas para esta fase de la investigación, en la cual se organizaron capítulos según cada objetivo, ya que corresponden al análisis de cada parte del sistema comunicativo de las estrategias: fuente - emisor - canal - mensaje - receptor. 


\section{Las estrategias de comunicación de los emisores: alcaldías de Circasia (Quindío) y Donmatías (Antioquia)}

Las estrategias de comunicación forman parte del sistema de comunicación pública (SC), en la teoría de producción social de comunicación de MartínSerrano (1986), ya que son planeadas y ejecutadas por las alcaldías con el uso de medios, canales y redes de comunicación. Es importante describir cómo fue la planeación y ejecución de las estrategias durante el aislamiento preventivo obligatorio, de ahora en adelante mencionado como APO, a través del método estudio de caso, con base en la postura de los autores Escudero, Delfin y Gutiérrez (2008), quienes definen su corte cualitativo, el cual permite el análisis y observación de un fenómeno en situaciones específicas.

Su principal objetivo es la descripción para detectar características y contextos del objeto de estudio. En desarrollo del estudio de caso se realizaron cuatro entrevistas semiestructuradas por medio de la plataforma Meet a los funcionarios de las alcaldías: jefe de Comunicaciones de la Alcaldía de Donmatías, Diego Alejando Gómez Gómez; jefe de Comunicaciones de la Alcaldía de Circasia, Lina María López Jiménez; directora del Plan Territorial de Salud de Circasia, Mayra Benítez Dorado; secretaria de Salud, Laura Andrea Cataño López.

Martín-Serrano, en la teoría social de la comunicación (1986), señala que hay afectaciones mutuas entre el sistema de comunicación y el sistema social porque ambos comparten una superestructura, una estructura y una infraestructura; alli son necesarios los recursos materiales, el apoyo y acompañamiento de las personas que mantengan una relación de interdependencia: “... algunos cambios sociales explicarán algunas transformaciones de la comunicación pública, y que al mismo modo, algunas transformaciones comunicativas tuviesen a veces algunas consecuencias para la estructura y el funcionamiento de la sociedad" (1986, p. 60). Es el caso de los cambios producidos por la pandemia, puesto que la sociedad estaba acostumbrada a realizar sus procesos de manera presencial, a causa del brote de la COVID-19 migraron a lo digital para satisfacer sus necesidades individuales y colectivas de salud.

Se halló que en ninguna de las dos administraciones existen diagnósticos sobre las necesidades de información y comunicación de sus públicos de interés. Como consecuencia, realizaron ejercicios informales con base en su experiencia porque la mayoría de sus procesos funcionaron según las circunstancias que se presentaron en el entorno; la mayoría de sus estrategias digitales migraron y crearon un directorio digital de los establecimientos para la reactivación económica. Las estrategias se definen como...
Comunicación

número 46

Enero-junio

2022
1 Para Martín-Serrano (1986), la superestructura se define en el sistema social como normas, ideas y creencias; $y$ en el sistema de comunicación pública, es la visión de lo que acontece propuesta en las narraciones. La estructura, en el sistema social, como organizaciones para la producción y reproducción en la sociedad y, en el sistema de comunicación pública, organizaciones mediadoras. La infraestructura, en el sistema social, como recursos y equipamiento para la producción y reproducción social, y, en el sistema de comunicación pública, como medios de producción, difusión y recepción de la información. 
... un conjunto de acciones que se llevan a cabo para lograr un determinado fin que asegura una decisión óptima en cada momento, cuyo objetivo prioritario es incrementar los niveles de impacto y la efectividad tanto del comportamiento individual como el cambio social y organizacional (De Lorenzo, 2014, p. 21).

En este sentido, las estrategias de comunicación forman parte de la estructura del sistema de comunicación pública de las alcaldías de Circasia y Donmatías, puesto que se dedicaban a mediar entre la institución y la sociedad civil en aquellos aspectos relevantes para la prevención y promoción frente a la COVID-19. Se describen las estrategias de comunicación utilizadas durante el APO.

Circasia diseñó siete:

Imagen 1. Campaña de prevención ante la COVID-19 de la Alcaldía de Circasia entre marzo y agosto de 2020.

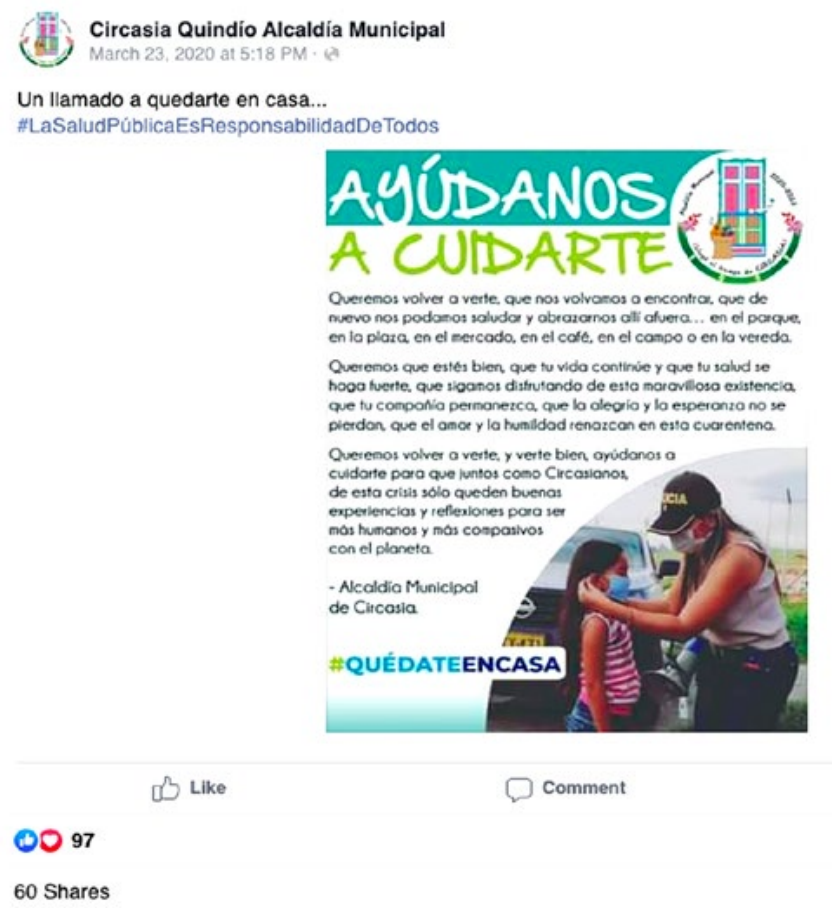

Fuente: Alcaldía Municipal de Circasia (2020a).

1. Acciones informativas de la administración municipal relacionadas con la COVID-19. Planteadas con el objetivo de informar a los públicos externos por medio de tácticas en torno a la prevención. 
2. Campañas internas. Dirigidas a los públicos internos de la alcaldía para informarles a los funcionarios acerca de las acciones implementadas durante la contingencia de la COVID-19.

3. Replicadores de la información. Los funcionarios públicos cumplieron el papel de ser un nodo-red de multiplicadores de la información a través de WhatsApp y Facebook.

4. Atención COVID-19. Contenido sobre los riesgos de salud pública provocados por la pandemia en los procesos presenciales y virtuales.

5. Donatón. ${ }^{2}$ Esta estrategia se materializó en una campaña para sensibilizar a los circasianos sobre la ayuda a personas necesitadas.

6. Reapertura económica. Acompañamiento y promoción a los establecimientos de comercio para realizar la apertura económica cumpliendo los protocolos de bioseguridad compartidos a través de boletines, afiches y redes sociales.

7. Free press. Uso de plataformas para envío de correos masivos, con el fin de crear un canal de comunicación entre los periodistas y la administración para informar en medios locales y departamentales.

Las estrategias utilizadas por la Alcaldía de Donmatías durante el APO fueron:

1. Programa institucional Donmatías Imparable. Realizado en La Voz de Donmatías y Teledonmatías para dar a conocer los procesos de la administración municipal y datos relevantes de la contingencia de la COVID-19.

2. Acciones informativas presenciales La Alcaldía Más Cerca. Para explicarle a la comunidad las medidas de autocuidado e informar a la población sin acceso a medios tradicionales o digitales.

3. Quédate en Casa. Estrategia integral realizada para la prevención del contagio de la COVID-19 a través de la generación de contenidos en formatos audiovisuales para redes sociales.

4. Autocuidado de la salud y reactivación económica. Publicó en redes sociales diferentes campañas con el fin de generar cultura de autocuidado, sentido de pertenencia y concientización entre dueños de establecimientos de comercio y comunidad en general.

5. Pedagogía. Para atender las medidas y consecuencias de la COVID-19 desde una perspectiva social, creó contenidos para que los ciudadanos encontraran una línea de atención para escuchar sus dificultades. ${ }^{3}$

6. Donatón. Esta estrategia fue materializada en varios eventos virtuales, con el propósito de sensibilizar a los donmatieños sobre la ayuda a personas necesitadas. ${ }^{4}$

7. Free press. Con el apoyo de los medios aliados, la alcaldía creó contenidos para publicar en medios locales, regionales y nacionales acerca de las medidas tomadas por la administración.
2 Recibieron un total de 298 kits de viveres, gracias a la solidaridad de los circasianos.

3 La alcaldía puso a disposición sus psicólogos para atender los llamados de la ciudadanía, y creó contenidos en los cuales los profesores apoyaban la labor de los padres de familia en el proceso académico de sus hijos y compartían reflexiones sobre la importancia de la tolerancia y el respeto en el círculo familiar.

4 La Donatón tuvo como resultado, entre especie y dinero, un total de 178.118 .050 pesos en Donmatías. 


\section{Imagen 2. Medidas de prevención tomadas}

por la Alcaldía de Donmatías.

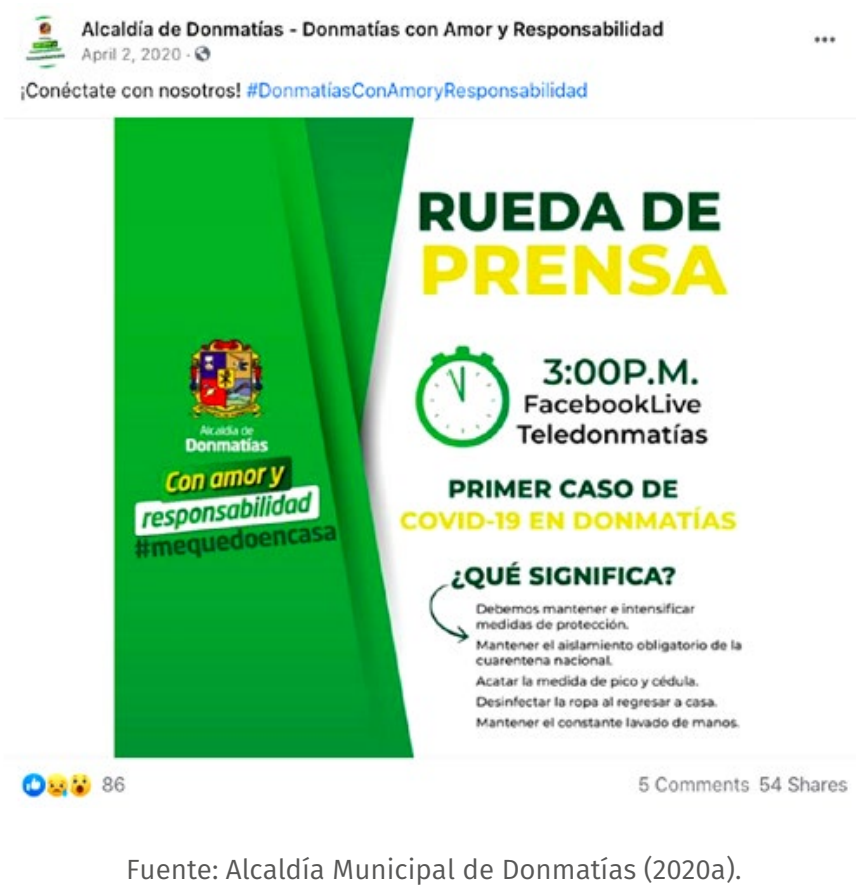

En el marco de las organizaciones públicas, las teorías estratégicas de la comunicación encuentran un campo de acción que requiere enfoques específicos en el modelo, con énfasis en la gobernanza y el diálogo para la búsqueda del consenso en los públicos. La información adecuada y oportuna da legitimidad a las instituciones; por consiguiente, la estrategia de comunicación consiste en la capacidad de gestionar mensajes y significados dentro de un proceso de interacción con el objetivo de persuadir a los interlocutores (Preciado Hoyos y Guzmán Ramírez, 2012).

Así pues, las estrategias parten de situaciones específicas analizadas para construir un escenario donde se dan dos cambios fundamentales en su adaptación. El primero prioriza aspectos relacionales que indaguen las necesidades de la comunidad con un sentido humano. El segundo comprende la transformación desde el ámbito social, partiendo de un diálogo entre el emisor y el receptor en una perspectiva multidimensional.

Según la descripción de las estrategias de comunicación implementadas y con base en los resultados de las entrevistas semiestructuradas, se aprecia que las dos administraciones llevan a cabo los procesos con enfoque operativo, basado en las actividades, por cuanto carecen de un diagnóstico y de un plan que permita tener estrategias de comunicación y no solo actividades. 
Así las cosas, se hace necesaria la comunicación estratégica, la cual, según De Lorenzo (2014), “provee de un 'paraguas conceptual' que ofrece a las organizaciones la posibilidad de integrar sus esfuerzos comunicacionales, que de otra manera se encontrarán dispersos e inconexos" (2014, p. 8). Por esta misma causa, no se tienen segmentados los públicos, pues hay poco conocimiento de sus características sociodemográficas, culturales y económicas, y de sus necesidades de información y comunicación.

\section{Canales y medios de comunicación de las alcaldías de Circasia (Quindío) y Donmatías (Antioquia)}

El estudio de los canales y medios existentes en las alcaldías permitió revisar el contexto en el que se desarrollan las estrategias de comunicación mencionadas anteriormente. Con el fin de reconocer las características de los canales y medios, se utilizó el estudio de caso, método útil para distinguir las particularidades de cada administración.

La de Circasia cuenta con los siguientes medios y sus respectivos canales de comunicación para la difusión de sus estrategias:

1. Mailchimp. Es una plataforma de envío masivo de correos electrónicos; canal utilizado por la Oficina de Comunicaciones para redactar boletines de prensa dirigidos a los contactos sistematizados en bases de datos.

2. Medios tradicionales de comunicación. La Oficina de Comunicaciones, a través de su estrategia de Publicity, busca la publicación de su contenido informativo en los canales regionales y locales.

3. WhatsApp. Canal de comunicación que facilita la rápida difusión de los mensajes y la conformación de nodos de información entre los funcionarios de la administración municipal y la comunidad en general.

4. Instagram.Laadministración municipaltieneelperfil@alcaldiadecircasia, que funciona como un canal de comunicación.

En Donmatías utilizan los siguientes medios y respectivos canales de comunicación para la difusión de sus estrategias:

1. Televisión. Tiene dos programas: el programa institucional y microprogramas con las diferentes dependencias, además de pauta institucional incluida en su parrilla cotidiana en Teledonmatías.

2. Radio. La emisora local emite un programa en directo para el diálogo con el alcalde por ser el medio de más amplio acceso y difusión, principalmente en la zona rural ${ }^{5}$ del municipio.
5 Dentro del área territorial rural de Donmatías, hay 16 veredas y un corregimiento. 
3. Comunicación interpersonal. Continúa siendo el medio por excelencia: el contacto físico con el otro para llegar a los lugares de más dificil acceso tecnológico dentro del municipio.

4. Redes sociales. La administración municipal usa como medios Instagram (@camilocorreaalvarez), YouTube (@comunicacionesdonmatias) y Twitter (@DonmatiasAlc), con el fin de aprovechar sus diferentes formatos y audiencias.

Hay medios y canales que las dos administraciones tienen en común:

1. Líneas telefónicas. Este medio les sirve para entablar una comunicación directa con la ciudadanía sobre temas específicos.

2. Altavoces. El perifone $0^{6}$ es un canal que genera una cercanía con la comunidad al llegar a cada barrio para informar las medidas tomadas.

3. La web. El ciudadano puede encontrar información oficial de primera mano, además de hacer trámites en las páginas oficiales: www.circasiaquindio.gov.co y www.donmatías-antioquia.gov.co

4. Facebook. Es una de las redes sociales que los jefes de comunicaciones posicionan como de mayor importancia para conectarse con la comunidad, por cuanto ofrece configuraciones y formatos para las publicaciones desde los perfiles: Alcaldía de Donmatías - Donmatías con Amor y Responsabilidad y Circasia Quindío Alcaldía Municipal.

Estos canales y medios son un factor relevante porque sirven para informar e influyen y fomentan las políticas públicas orientadas a la salud. Según la Organización Mundial de la Salud (OMS) (2018), "la comunicación de riesgos consiste en el intercambio, en tiempo real, de información, consejos y opiniones entre los expertos, los líderes comunitarios, los funcionarios públicos y las personas en situación de riesgo".

Facebook es el medio en el que más interactúa la comunidad con las dos administraciones, pues es una plataforma que permite compartir textos, fotos, videos, transmisiones en vivo, y que sirve como convergencia de otros canales de comunicación.

De acuerdo con Piñuel Raigada (2012):

Los medios disponibles resultan ser herramientas o útiles biológicos y/o tecnológicos de acomodación frente al entorno, asi como dimensiones en virtud de las cuales los medios disponibles se convierten en medios de producción de bienes o servicios comunicativos (con un valor social de uso y de cambio) (2012, p. 140).

Según la aplicación de un cuestionario en los dos municipios (Gráfica 1), Facebook trasciende la dimensión de utilidad y se halla dentro de la
6 "Transmitir por medio del teléfono sin hilos una pieza de música, un discurso o una noticia en condiciones determinadas y a hora fija" (Real Academia Española [RAE] y Asociación de Academias de la Lengua Española [Asale], 2014). 
dimensión de acomodación al entorno. Esta red social es una de las más visitadas y los ciudadanos la clasifican como medio oficial.

\section{Gráfica 1. Interacción de los ciudadanos con medios y canales.}

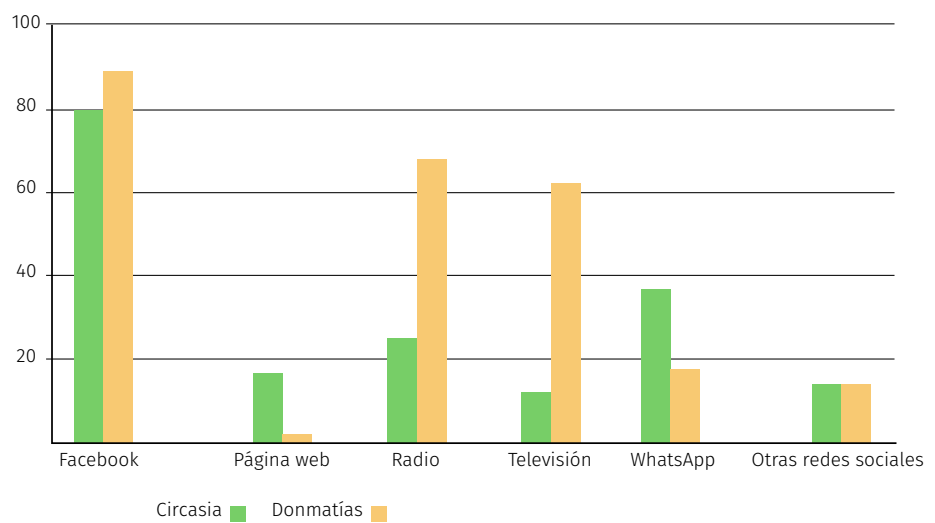

Fuente: Elaboración propia.

En esta red social se presenta otro fenómeno interesante para abordar: en la Alcaldía de Circasia se destaca la institucionalidad de la administración en su propio perfil, dado que comunica con un tono riguroso y comparte contenido netamente institucional. En cambio, Alcaldía de Donmatías lo hace de forma interpersonal por medio de la figura del alcalde; entonces, se observa mayor interacción en las páginas personales de Camilo Correa Álvarez, quien se asimila, así, a la figura de líder carismático (Weber, 2002), que se adapta y conecta en todos los contextos con los diferentes escenarios de la comunidad, generando una comunicación efectiva como un propósito fundamental. El alcalde del municipio logró una conexión constante con los ciudadanos y mayor interacción en sus redes sociales, lo cual lo convirtió en animador de políticas culturales y sociales y generó una modificación en el sistema institucional de comunicación.

Así pues, en esta investigación se hallaron dos caminos utilizados por las administraciones para lograr dicha legitimidad: el primero, en Donmatías, impulsa a través de los medios masivos de comunicación la imagen del alcalde para legitimar a la administración y sus estrategias; y el segundo camino, utilizado en Circasia, donde la institucionalidad de la propia administración legitima las acciones propias. 
Imagen 3. Comparación de seguidores en perfiles de Facebook.
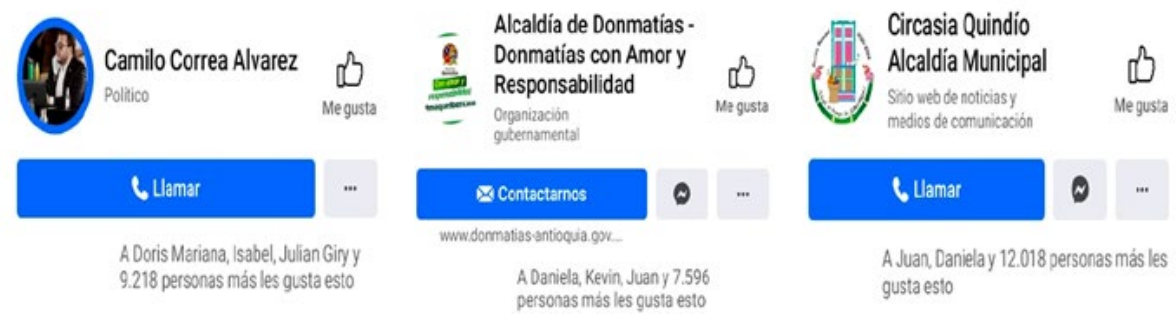

6 Llamar

0

A Juan, Danielay 12.018 personas más les gustaesto

Se destaca el hallazgo hecho sobre la Alcaldía de Donmatías: la figura de su alcalde goza del reconocimiento de sus ciudadanos desde las estrategias de comunicación, las cuales, en el ámbito personal y no institucional, le dan a este dirigente un carácter carismático, en sintonía con las teorías de Max Weber (2002) (actualizadas por López Zafra, 2001; Piñuel Raigada, 2012; Serrafero, 2018). Estas postulan tres tipos de autoridad, y en el caso en cuestión estaría esta basada en lo que el autor denomina democracia plebiscitaria, contraria a la democracia parlamentaria, ya que la primera es un tipo de dominación carismática. El resurgimiento del liderazgo carismático se produce porque este viene con mayores beneficios para los proyectos personales de los dirigentes; y como se ve en los resultados de la investigación, dichos proyectos dependen de la relación carismática entre el líder y sus seguidores, concebida primero como una propiedad y luego como un proceso.

\section{Mensajes para la promoción de la salud y la prevención de la COVID-19 en las alcaldías de Circasia (Quindío) y Donmatías (Antioquia)}

Para reconocer los mensajes y sus funciones se utilizó el método de análisis de contenido, que posibilita la observación y el estudio de diferentes contenidos de comunicación relevantes para cada proceso (Galeano, 2018). Su principal característica es que revela aquellos aspectos que van más allá de lo textual o expresado, es decir, el contenido latente, en este caso, de las publicaciones de las alcaldías de Circasia y Donmatías entre marzo y agosto de 2020.

En un primer momento, se definió el objeto de estudio, corpus textual recopilado de las páginas oficiales de Facebook (Alcaldía de Donmatías Donmatías con Amory Responsabilidad y Circasia Quindío Alcaldía Municipal), dado que, según expresan los comunicadores de ambas organizaciones, este es el medio más eficiente para que los ciudadanos se informen. Por esta razón, se eligió la popular red social, y se seleccionaron dos publicaciones 
semanales, para un total de 23 por municipio, entre los meses de marzo y agosto de 2020, considerando criterios como el número de reacciones, interacciones y que su contenido tuviera relación con la COVID-19.

Con base en los textos publicados, los fragmentos de video, de audio y las piezas gráficas, se definió como unidad de registro a la frase, entendiendo por el término frase una unidad textual con sentido completo. Según la agrupación de frases recopiladas de ambas alcaldías se buscaron las categorías de segundo orden, también llamadas categorías emergentes.

\section{Categoría: Estrategias de comunicación}

1. Relacionamiento con los medios. Se encontró que los mensajes de relacionamiento con los medios utilizan un lenguaje formal y un tono informativo para hablar de asuntos claves durante ruedas de prensa.

2. Identificación de públicos. Dado que son municipios con poca cantidad de habitantes y que, por ello, los alcaldes pueden dar mensajes directos, cara a cara, hablar con su gente en el espacio público para hacer pedagogía, se encontró que los mensajes coloquiales adquieren un tono impositivo con el fin de llamar la atención sobre los cuidados que deben tener los ciudadanos; a la vez, hacen referencia a acciones que las administraciones van a ejecutar.

3. Presencialidad. Los mensajes presenciales fueron utilizados para informar sobre protocolos de bioseguridad, apertura económica, decretos, entre otros, en las poblaciones donde es dificil el acceso a internet o donde no tienen conocimiento de estos procesos digitales. Las administraciones usaron aquí cifras y datos en un tono racional cuantitativo.

4. Digitalidad. Para reactivar la economía e impulsar la compra local en los municipios, crearon piezas gráficas con información de contacto y especificaciones de los establecimientos de comercio propios; esta estrategia llamaba la atención de los ciudadanos por el lenguaje cercano y porque apelaba a la emoción y sentido de pertenencia con los productos y servicios locales. 
Imagen 4. Telecafé Noticias (2020).
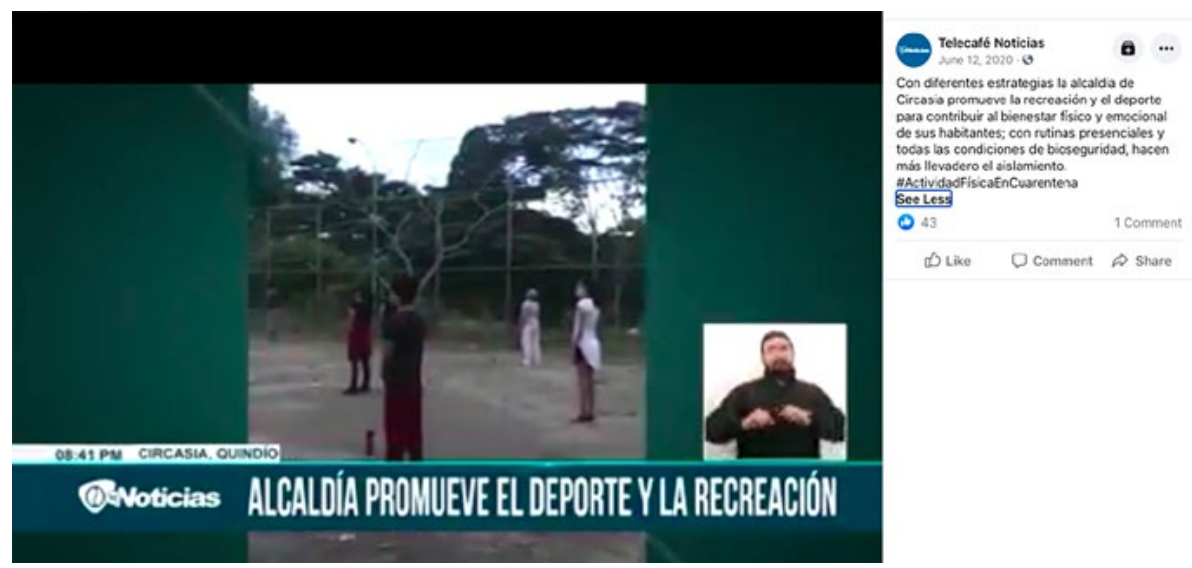

\section{Categoría: Comunicación pública}

1. Informar. Las redes sociales, Facebook sobre todo, fueron fundamentales para la fácil divulgación de noticias, decretos, líneas de atención y reportes, por medio de mensajes formales que transmitían confiabilidad. Durante la contingencia, las instituciones estatales fueron el principal medio de información para la comunidad sobre decisiones para prevenir y mitigar el contagio del virus.

2. Educar. Durante el período estudiado, se observó un enfoque educativo para transmitir mensajes sobre cómo implementar los protocolos de bioseguridad adecuadamente, sobre el manejo de emociones y sobre la buena convivencia (entre otros, sobre la tolerancia y respeto al personal de salud que estaba al frente de esta pandemia). Las estrategias de comunicación buscaban innovar y concientizar para mover fibras en sus comunidades con un tono emotivo y cercano.

\section{Categoría: Crisis}

1. Inmediatez. No había un manual de crisis para la situación que se presentó; así que debieron crear estrategias de comunicación inmediatas, para mantener la gobernanza y la ejecución de proyectos en su período administrativo, con el fin de dar a conocer la importancia de reforzar las medidas de autocuidado y la necesidad de tomar decisiones arbitrarias que afectaran la vida cotidiana de los ciudadanos, todo con el uso de cifras para dimensionar los riesgos.

2. Impacto. Esta crisis generó necesidades en las comunidades, sobre todo en las más vulnerables al contagio, como los adultos mayores; por ello, fue importante que las alcaldías atendieran estos públicos en diferentes 
aspectos transversales a las dimensiones de los ciudadanos: lo cultural, personal, económico, físico y político, con el fin de generar bienestar.

\section{Categoría: Comunicación en salud y salud pública}

1. Institucionalidad. Fue necesario hacer uso de los mensajes para reforzar la imagen institucional de ambas alcaldías y sus alcaldes para lograr legitimidad; en algunas ocasiones, recurrieron al uso de conjugaciones verbales en primera persona y a referencias a la labor del equipo de trabajo en sus actividades.

2. Creación de contenidos. Generar contenidos multimediales que informen y eduquen a la comunidad desde la comunicación en salud fue una de las tácticas ejecutadas en el marco de las estrategias de las alcaldías contra la COVID-19. Un lenguaje carismático para que la comunidad se sintiera identificada y reflexionara sobre la importancia de cuidar la vida de los familiares y de la comunidad.

Figura 5. Contenido generado por la Alcaldía de Donmatías.
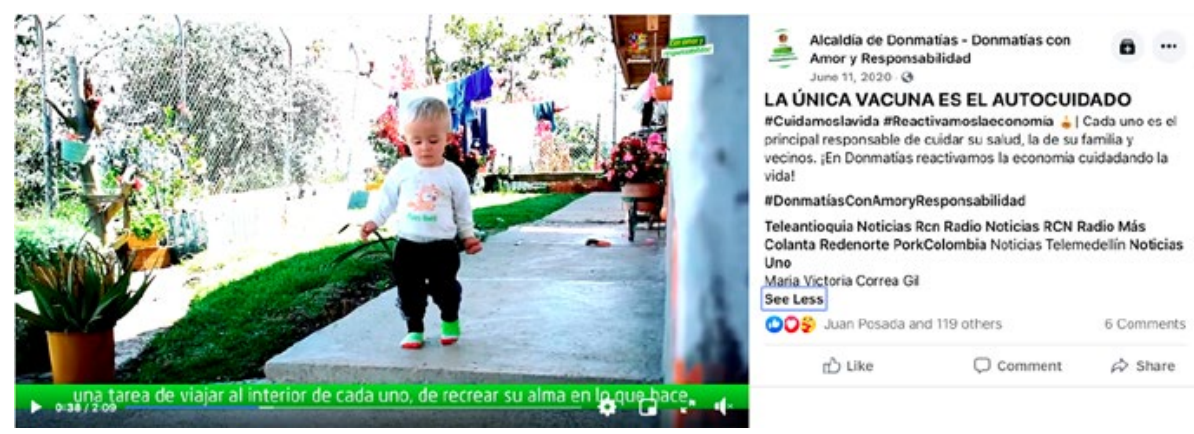

Fuente: Alcaldía Municipal de Donmatías (2020b).

De acuerdo con las funciones clásicas de los medios de comunicación (informar, entretener, educar y generar opinión), se muestra que los cambios repentinos en los procesos de comunicación son "la representación de lo que cambia en la comunicación pública con lo que se transforma en la sociedad" (Martín-Serrano, 1986, p. 79). Así se permite articular las relaciones de información, organización y acción social que influyen en el comportamiento de las personas; en este caso, se hace referencia al $\mathrm{APO}$, que generó cambios obligatorios en la rutina de los colombianos y la necesidad de que permanecieran en contacto con las administraciones para estar enterados sobre la contingencia. 


\section{Gráfica 2. Intención de visita a los medios de las alcaldías.}

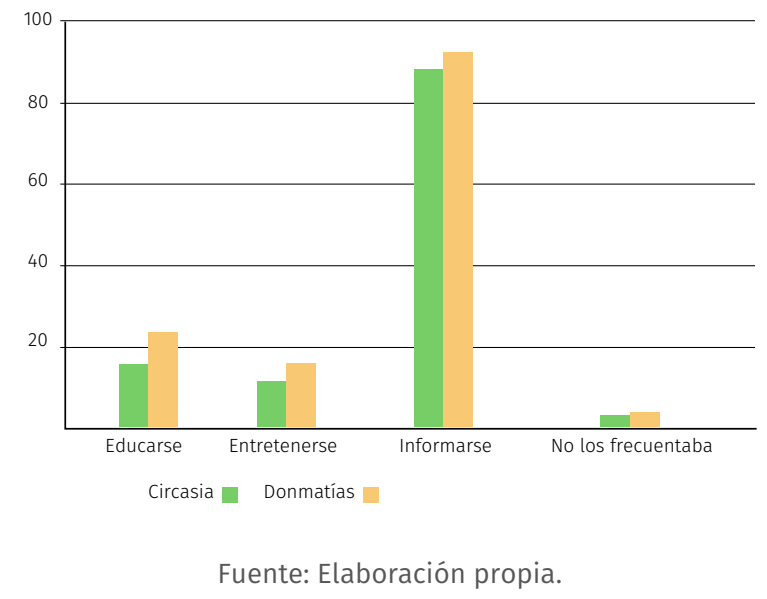

A partir de los tres métodos de investigación se puede comprender que ambas oficinas de comunicaciones implementaron un lenguaje persuasivo para difundir los mensajes: "Queremos volver a verte, y verte bien, ayúdanos a cuidarte para que juntos como circasianos, de esta crisis solo queden buenas experiencias y reflexiones para ser más humanos y más compasivos con el planeta" (Alcaldía Municipal de Circasia, 2020b).

Sin embargo, en momentos críticos del APO, para ambas administraciones fue necesario hacer uso del lenguaje impositivo con la vocería de los alcaldes, con el fin de garantizar el cumplimiento de los decretos del Gobierno nacional, que exigían una respuesta inmediata.

Al cruzar la información obtenida en las entrevistas con los datos de los cuestionarios, se encontró la coincidencia de ambas administraciones en el esfuerzo para que sus mensajes fueran comprendidos por la población en general. Se resalta que la comunidad recuerda en mayor medida los mensajes relacionados con el autocuidado, la responsabilidad y la importancia de permanecer en casa para evitar aglomeraciones como acciones fundamentales para enfrentar la crisis de la COVID-19, haciendo énfasis en el deber individual de cuidar la salud pública.

\section{Comprensión de los mensajes por parte del receptor}

Terminado el proceso de revisión de las estrategias de comunicación, se procedió a examinar el grado de comprensión de los mensajes a través del método de análisis estadístico de la población, que permite organizar, 
sintetizar, ver sus características más relevantes y presentar la información de una forma concisa y clara. Se examinaron los datos con el uso de los Formularios Google, herramienta para aplicar y obtener los resultados del cuestionario, que constó de 14 preguntas.

A través de grupos de WhatsApp institucionales, administrados por funcionarios, y comunitarios, creados por la sociedad en general, se invitó a diligenciar y compartir con los contactos el cuestionario, para lograr un muestreo en bola de nieve que dio como resultado la respuesta del 19,2 $\%$ de los integrantes de los grupos: 109 donmatieños y 105 circasianos. En promedio, un $82,3 \%$ de ellos residían en el sector urbano y un $15 \%$ en el sector rural.

\section{Gráfica 3. Edades de los participantes en el cuestionario.}

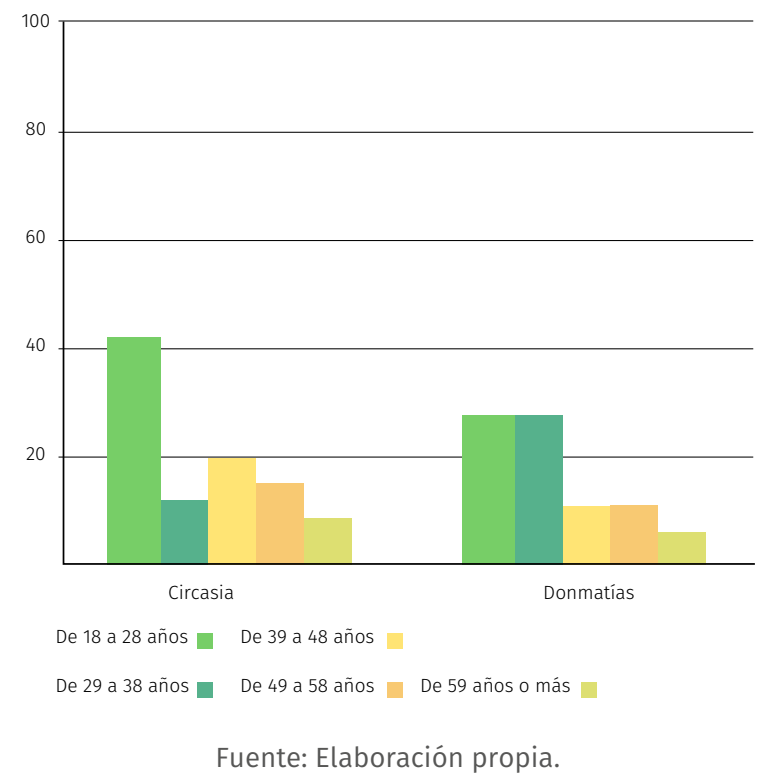

Las estrategias de comunicación para la promoción y prevención en salud pública frente a la COVID-19 fueron recibidas en mayor medida por personas del sector urbano ubicadas en una edad entre los 18 a 28 años, que representan en Circasia un $41 \%$ y en Donmatías un 33 \%. En ambos municipios, las franjas minoritarias tienen 49 años o más, lo que relaciona a personas de mayor edad con un menor acceso a la red en la cual se divulgó el cuestionario.

Según la Organización Mundial de la Salud (1986), "la promoción de la salud exige la acción coordinada de todos los implicados: los gobiernos, 
los sectores sanitarios y otros sectores sociales y económicos, las organizaciones benéficas. Las autoridades locales, la industria y los medios de comunicación". Por ello, fue importante examinar la comprensión de los mensajes en cada una de las comunidades, con el fin de conocer su opinión sobre las estrategias de comunicación implementadas por las alcaldías, y así continuar generando procesos coordinadamente.

Dicha acción necesita una constante relación entre las administraciones y los ciudadanos. Para esta investigación, ello se demuestra en los resultados obtenidos por el cuestionario: el 41,5\% de los participantes de Donmatías dijeron que la comprensión de los mensajes fue muy alta, en contraste con Circasia, donde fue del $14 \%$. Sin embargo, hubo una coincidencia en señalar alto el grado de comprensión en los dos municipios, con un 49,3\%, como se muestra en la Gráfica 4:

\section{Gráfica 4. Grado de comprensión de los mensajes.}

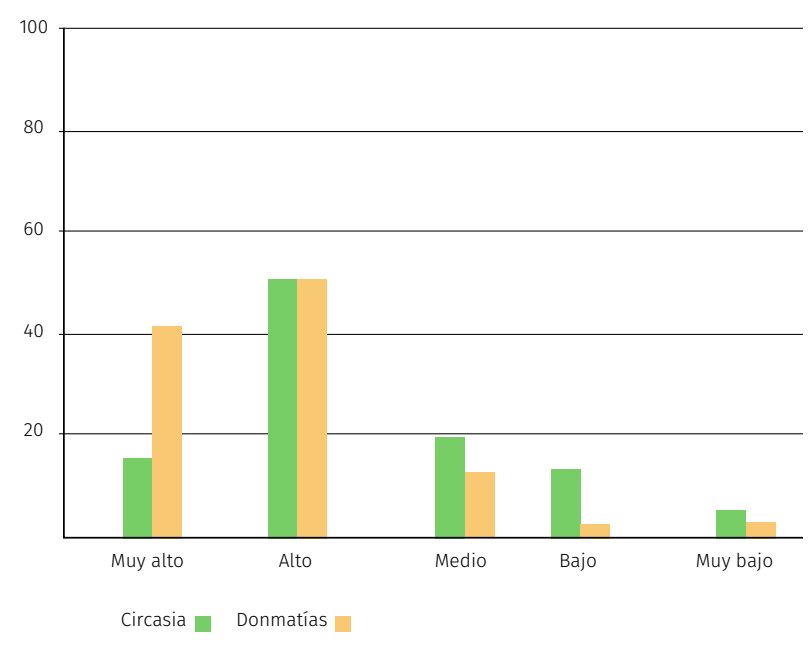

Fuente: Elaboración propia.

Las dos alcaldías estudiadas implementaron la comunicación en salud debido a la coyuntura que se apoderó de la agenda y como temática central en un proceso de participación. Para Blanca Deusdad (2003):

El líder político tiene que tener la capacidad de comunicar. Sus palabras deben conectar con el ciudadano medio, sus maneras deben ser vividas como propias; él debe pasar a ser considerado "uno de los nuestros". Así, el líder carismático comparte una misma identidad o varias con los seguidores y eso favorece la adquisición de carisma (2003, p. 26). 
Finalmente, es importante tener en cuenta que se deben comprender las estrategias de comunicación internamente, es decir, aquellas dirigidas a los colaboradores de las alcaldías, para que la comunidad en general entienda los mensajes y lograr un mayor alcance, pues los funcionarios constituyen una red de multiplicadores. Este es uno de los factores que garantizan la comprensión afirmada dentro del sondeo.

De Lorenzo (2014) afirma:

Es fundamental, desde el punto de vista de la dirección, que se generen y potencien las interacciones, o más bien lazos entre los sujetos pertenecientes a la organización. Esto es tan esencial como las relaciones establecidas con los públicos externos, ya que ayuda a generar una cultura de comprensión e implementación de la estrategia dentro de la empresa (2014, p. 37).

Un ejemplo está en la Alcaldía de Circasia: sus colaboradores se convirtieron en difusores, es decir, amplificaron la información, y están proyectados en la comunidad para fortalecer las diferentes estrategias de comunicación que emiten las dependencias.

\section{Reflexiones}

El modelo de estudio aquí realizado puede ser ejecutado en otros lugares $y$ en otros momentos de la pandemia, aunque posiblemente arroje resultados distintos, asociados a la evolución del fenómeno. Se presentan las conclusiones de la investigación:

Con el fin de describir las estrategias de comunicación, se crearon matrices para sistematizar la información suministrada en el trabajo de campo (variaron debido a ajustes necesarios durante el proceso). Se encontraron siete estrategias en cada una de las alcaldías, definidas sobre la marcha, tal como se evidenció en las entrevistas a los jefes de comunicaciones.

Se determinó la existencia de los canales y medios utilizados por las alcaldías de Circasia y Donmatías, en el marco de las estrategias para la comunicación con los ciudadanos durante la contingencia provocada por la COVID-19, entre marzo y agosto de 2020. Se vio que las dos alcaldías tienen a su disposición canales y medios de comunicación, utilizados durante dicha contingencia de la misma forma que lo hacían antes de ella: intuitiva o informalmente. Motivados por la pandemia y la necesidad de informar permanentemente, tuvieron un uso mayor de Facebook, como se muestra en la Gráfica 1 (en perfiles institucionales y personales de cada alcalde). Además, esta red fue la más usada por los ciudadanos de los dos municipios. 
Se reconocieron los mensajes que formaron parte de las estrategias usadas por las alcaldías para la promoción de hábitos necesarios ante la pandemia, entre marzo y agosto de 2020; los mensajes coincidieron en mantener un enfoque no prohibitivo, sino exhortativo e informativo. Realizar el análisis de contenido en la red social Facebook permitió tener acceso a la mayoría de los mensajes publicados en otros medios y canales donde convergen.

Se examinó el grado de comprensión de los mensajes de las estrategias comunicativas de ambas alcaldías durante la contingencia. Los ciudadanos participantes en la investigación manifestaron haber comprendido en alto grado los mensajes contenidos en las estrategias dirigidas a ellos en ambos municipios. Para este efecto, se diseñó un cuestionario en Google, que fue compartido en los grupos de difusión y contactos de WhatsApp de las dos alcaldías, lo cual facilitó que este llegara a un porcentaje significativo de personas.

Este estudio, en fin, ayuda a apreciar que para que exista una buena gestión y planeación de estrategias de comunicación, técnicamente planteadas, es necesario un diagnóstico sobre la comunicación y los factores que esta aborda, como los receptores, sus características y sus necesidades. En la página web comunicación en pandemia están los recursos interactivos que complementan este informe.

\section{Referencias}

Alcaldía Municipal de Circasia. (2020a). [Fan Page]. Facebook. https://www.facebook.com/ circasiaquindio.alcaldiamunicipal

Alcaldía Municipal de Circasia. (2020b, marzo 23). Un llamado a quedarte en casa. [Póster] [Actualización de estado]. Facebook. https://m.facebook.com/circasiaquindio. alcaldiamunicipal/posts/2460662477530900

Alcaldía Municipal de Donmatías. (2020a, abril 2). Rueda de prensa [Actualización de estado]. Facebook. https://www.facebook.com/DonmatiasConAmorYReponsabilidad/ posts/1513817922125909

Alcaldía Municipal de Donmatías. (2020b). La única vacuna es el autocuidado [Video] [Actualización de estado]. Facebook. https://www.facebook.com/ watch /?v=562516367798668

Aleixandre-Benavent, R., Castelló-Cogollos, L., \& Valderrama-Zurián, J.-C. (2020). Información y comunicación durante los primeros meses de Covid-19. Infodemia, desinformación y papel de los profesionales de la información. Profesional De La Información, 29(4). https://doi.org/10.3145/epi.2020.jul.08

Agudelo, A. (2016). La formación ciudadana a través de los medios de comunicación. La televisión colombiana y su aporte a las ciudadanías en salud. En El aporte de la televisión colombiana a la formación ciudadana en salud. Universidad de Antioquia. 
Bianco, A. y Martínez, E. (2004). Etapas de una investigación. Universidad de Buenos Aires. https://www.dm.uba.ar/materias/probabilidades_estadistica_C/2004/1/PyEC12.pdf

Castelló Cogollos, L., Valderrama, J. C., y Benavent, R. (2020). Información y comunicación durante los primeros meses de COVID-19. Infodemia, desinformación y papel de los profesionales de la información. Profesional de la Información, 29(4), 1-17. https://doi. org/10.3145/epi.2020.jul.08

De Lorenzo, M. (2014). Comunicación estratégica vs. estrategia de la comunicación. Las dos caras de una misma moneda [Trabajo de grado]. Universidad Rey Juan Carlos, España. http:/ / hdl.handle.net/10115/12229

Deusdad, B. (2003). El concepto de liderazgo político carismático: populismo e identidades [PDF]. Opción, 19(41), 9-35. https://www.redalyc.org/pdf/310/31004101.pdf

Escudero, J., Delfin, L. A., y Gutiérrez, L. (2008). El estudio de caso como estrategia de investigación en las ciencias sociales [PDF]. Ciencia Administrativa, (1), 7-10. https:/ / www.uv.mx/iiesca/files/2012/12/estudio2008-1.pdf

Galeano, M. E. (2018). Estrategias de investigación social cualitativa: el giro en la mirada. Universidad de Antioquia. http://hdl.handle.net/10495/11415

Galeano, M. E. (2018). Diseño de proyectos de investigación cualitativa. EAFIT. https://www. eafit.edu.co/cultura-eafit/fondo-editorial/colecciones/Paginas/diseno-de-proyectosen-la-investigacion-cualitativa.aspx

Hernández, Z. (2012). Métodos de análisis de datos: Apuntes [PDF]. Iberus. https://www. unirioja.es/cu/zehernan/docencia/MAD_710/Lib489791.pdf

López Zafra, E. (2001). ¿Liderazgo carismático en las organizaciones? Elementos para una reflexión sobre el cambio en las relaciones intraorganizacionales. Revista de Psicología Social, 16.

Martín-Serrano, M. (1986). La producción social de comunicación. Alianza.

Ministerio de Salud. (2020, mayo 26). Decreto 000844, por el cual se prorroga la emergencia sanitaria por el nuevo Coronavirus que causa la COVID-19 [PDF]. https://www.minsalud. gov.co/Normatividad_Nuevo/Resoluci\%C3\%B3n\%20No.\%20844\%20de\%202020.pdf

Ministerio de Salud, Oficina de Promoción Social. (2018). Estrategias de información, educación y comunicación en salud, incluyente y accesible [PDF]. https://www.minsalud. gov.co/sites/rid/Lists/BibliotecaDigital/RIDE/DE/PS/documento-estrategias-de-iec.pdf

Organización Mundial de la Salud [OMS]. (1986). Carta de Ottawa para la promoción de la salud [PDF]. https://www.paho.org/hq/dmdocuments/2013/Carta-de-ottawa-para-laapromocion-de-la-salud-1986-SP.pdf

Organización Mundial de la Salud [OMS]. (2018). Comunicación de riesgos en emergencias de salud pública [PDF]. https://apps.who.int/iris/bitstream/hand le/10665/272852/9789243550206-spa.pdf?ua=1

Piñuel Raigada, J. L. (2012). La noción de mediación comunicativa para el análisis y el diseño de la comunicación organizacional. Revista Icono14, 8(2), 125-152. https://doi. org/10.7195/ri14.v8i2.249

Preciado Hoyos, A. y Guzmán Ramírez, H. (2012). Gestión de la comunicación estratégica en los sectores empresarial, de desarrollo y público. Estudio Comparativo, 15(1), 128-159. https:// palabraclave.unisabana.edu.co/index.php/palabraclave/article/view/2071/2658 
Real Academia Española [RAE] y Asociación de Academias de la Lengua Española [Asale]. (2014). Diccionario de la lengua española.

Ríos Hernández, I. (2011). Comunicación en salud: conceptos y modelos teóricos.

Perspectivas de la Comunicación, 4(1), 5-30. http://revistas.ufro.cl/ojs/index.php/ perspectivas/article/view/111

Serrafero, M. (2018). Max Weber y la democracia plebiscitaria. Revista Internacional de Sociología, 76(2).

Telecafé Noticias. (2020). Con diferentes estrategias la Alcaldía de Circasia promueve la recreación y el deporte para contribuir al bienestar fisico y emocional de sus... [Video] [Actualización de estado]. Facebook. https://www.facebook.com/ watch / ?v=188653942499627

Weber, M. (2002). Economía y sociedad. Esbozo de sociología comprensiva. Fondo de Cultura Económica. 